\title{
Electromechanical Analysis of a Micromachined Comb-Drive Actuator by Admittance Measurement
}

\author{
Kenjiro Ayano Student Member (Kagawa University, s05d501@stmail.eng.kagawa-u.ac.jp) \\ Katsuyori Suzuki Student Member (Kagawa University, s02t423@stmail.eng.kagawa-u.ac.jp) \\ Gen Hashiguchi Member (Kagawa University, hasiguti@eng.kagawa-u.ac.jp) \\ Hiroyuki Fujita Member (The University of Tokyo, fujita@iis.u-tokyo.ac.jp)
}

Keywords : comb-drive actuator, Electromechanical Analysis, admittance measurement

\section{Introduction}

Electromechanical analysis of a micromachined comb-drive actuator based on equivalent circuit method is described. From the equivalent circuit, we formulated electrical admittance for a comb-drive actuator as a function of AC driving frequency and derived some relation between specific frequencies which can be obtained by admittance measurement. We have also found that mechanical parameters of a comb-drive actuator such as spring constant, rigid mass and mechanical resistance could be estimated by the relation of the specific frequencies. Using this theory we evaluated mechanical properties of a fabricated comb-drive actuator and confirmed its practical usefulness for characterization of a comb-drive actuator.

\section{Modeling of Comb Drive Actuator, and Formulization by Equal Circuit Method}

Fig. 1 shows a model of a comb-drive actuator to be analyzed, having a gap between opposing comb fingers of $\mathrm{d}$, structure height of $\mathrm{b}$, and initial overlap of comb fingers of $\mathrm{x}_{0} \cdot \mathrm{C}_{0}$ and $\mathrm{C}_{\text {stray }}$ are a total capacitance between opposing comb-drive fingers and a stray capacitance of the electrical system, respecitively. The actuator is supposed to be drived by $\mathrm{DC}$ bias $\mathrm{E}_{0}$ superposing small $\mathrm{AC}$ power

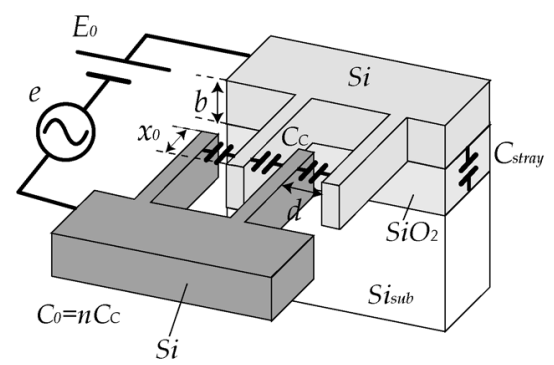

Fig. 1. Analysis model of a comb-drive actuator

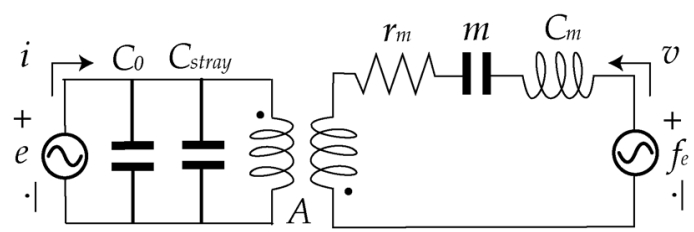

Fig. 2. The equivalent circuit of the comb-drive actuator e. The analysis is started from introduction of Lagragian $L$ for the comb-drive actuator as functions of velocity $\mathrm{v}$, displacement $\mathrm{x}$, and change q. The equivalent circuit can be drawn as Fig. 2 for the comb-drive actuator. As shown in Fig. 2, the parameter A is important factor to couple the electrical part with the mechanical part. If $\mathrm{DC}$ bias $\mathrm{E}_{0}$ is zero, the coupling doesn't occur, while for large $\mathrm{E}_{0}$ strong coupling is expected.

\section{Frequency Dependence on Admittance of a Comb-drive Actuator}

As described the previous section, electrical and mechanical circuits are coupled when DC bias $\mathrm{E}_{0}$ is not zero. Therefore, it is possible to observe mechanical motion by measuring electrical characteristics. The calculation result obtained from the theoretical formula is shown in Fig. 3. It turned out that the resonant frequency $\omega_{0}$ of a machine system exists between the frequency $\omega_{1}$ of zero point, equivalent to peak $\omega_{\mathrm{p}}$ of admittance, and found out that it was possible to perform machine characteristics evaluation of a comb-drive actuator.

\section{Conclusion}

When admittance was measured the actually created comb-drive actuator in LCR meter (H.P.4192A), as it was shown in Figure 4, the same graph as Figure 3 was obtained. It became clear theoretically from the relational expression of frequency and admittance that parameters, such as the mass $\mathrm{m}$ of a comb-drive actuator, mechanical resistance $\mathrm{r}_{\mathrm{m}}$, compliance $\mathrm{C}_{\mathrm{m}}$, and sharpness-of-resonance $\mathrm{Q}$, are computable based on this graph. A very useful thing is shown in the theory of this paper evaluating the mechanical parameter of a comb-drive actuator.
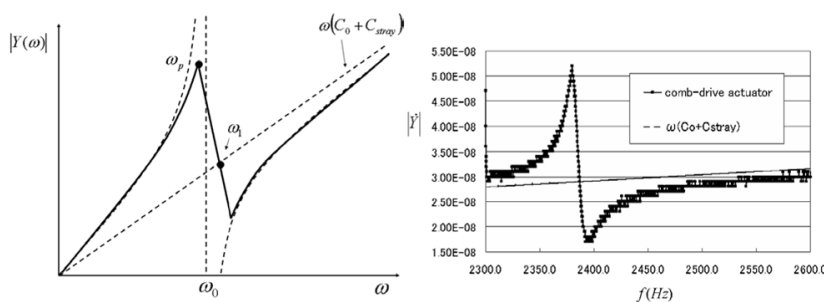

Fig. 3. Frequency depen- Fig. 4. Measured admittdence on absolute value of ance as a function of $\omega$ admittance 


\title{
Electromechanical Analysis of a Micromachined Comb-Drive Actuator by Admittance Measurement
}

\author{
Kenjiro Ayano* Student Member \\ Katuyori Suzuki* Student Member \\ Gen Hashiguchi* Member \\ Hiroyuki Fujita $^{* *}$ Member
}

\begin{abstract}
Electromechanical analysis of a micromachined comb-drive actuator based on equivalent circuit method is described. From the equivalent circuit, we formulated electrical admittance for a comb-drive actuator as a function of AC driving frequency and derived some relation between specific frequencies which can be obtained by admittance measurement. We have also found that mechanical parameters of a comb-drive actuator such as spring constant, rigid mass and mechanical resistance could be estimated by the relation of the specific frequencies. Using this theory we evaluated mechanical properties of a fabricated comb-drive actuator and confirmed its practical usefulness for characterization of a comb-drive actuator.
\end{abstract}

Keywords: comb-drive actuator, admittance measurement

\section{Introduction}

Comb-drive actuator is widely used in micro electro mechanical systems(MEMS) as a key element of actuation or sensing. From the view point of electromechanical transducers, comb-drive actuators is thought as a machine converting electrical energy into mechanical energy, and vice versa. In the case of electrostatic actuators, an electric field acts as medium coupling electrical power with mecahnical power; when a mechanical part is moving with a short distance $l$ in an electric field $E$, the electromotive force $V(=E l)$ will be appeared in the system, while when there are some electrical changes $q$ in an electric field $E$, the force $F=(q E)$ will act on the system.

The equivalent circuit method(1)(2), in which both the electrical and mechanical portions of the actuator are represented by electrical equivalents, is a useful method to analyze the behavior of electromechanical transducers. We formulated electrical admittance using the equivalent circuit method of a comb-drive actuator with taking account of stray capacitance of the electrical part and drew the admittance curve as a function of AC driving frequency. The graph shows a clear peak near mechanical resonant frequency if the stray capacitance is relatively small compared with the capacitance between comb-drive figures. By recent MEMS technology, especially progress of deep reacitive ion etching (DRIE)technique, we can easily obtain deep structures with small gap, thereby the device structure to observe such a peak have been realized.

\footnotetext{
* Faculty of Engineering, Kagawa University, 2217-20 Hayashicho, Takamatsu 761-0396

** Institute of industrial Science, The University of Tokyo,

4-6-1Komaba, Meguro-ku, Tokyo 153-8505
}

In this paper, we present an analysis method of the comb-drive actuator based on the equivqlent circuit method, and also show an estimation method of mechanical property including spring constant, moving mass, mechanical resistance by the admittance measurement.

\section{Modeling of Comb Drive Actuator, and Formulization by Equal Circuit Method}

Fig. 1 shows a model of a comb-drive actuator to be analyzed, having a gap between opposing comb fingers of $d$, structure height of $b$, and initial overlap of comb fingers of $x_{0} . C_{0}$ and $C_{\text {stray }}$ are a total capacitance between opposing comb-drive fingers and a stray capacitance of the electrical sysytem, respecitively. The actuator is supposed to be drived by DC bias $E_{0}$ superposing small AC power $e$. The analysis is started from introduction of Lagragian $L$ for the comb-drive actuator as functions of velocity $v$, displacement $x$, and change $q$.

$$
L=\frac{1}{2} m v^{2}-\frac{x^{2}}{2 C_{m}}-\frac{q^{2}}{2\left\{C(x)+C_{\text {stray }}\right\}} \cdots \cdots
$$

Where $m$ and $C_{m}$ are a rigid mass and a compliance of the comb-drive actuators, respectively, and $C(x)$ is a changing capacitance between opposing comb-drive figures.

In this cace, $C(x)$ is given by

$$
C(x)=\frac{2 n \varepsilon_{0} b\left(x_{0}+x\right)}{d},
$$

where $x$ is the small displacement of the comb-drive actuator, $\mathrm{n}$ is the number of comb-drive fingers, and $\varepsilon_{0}$ is the permittivity in vacuum.

Dissipation function $F$ is also given as functions of $\mathrm{v}$ and current i. 


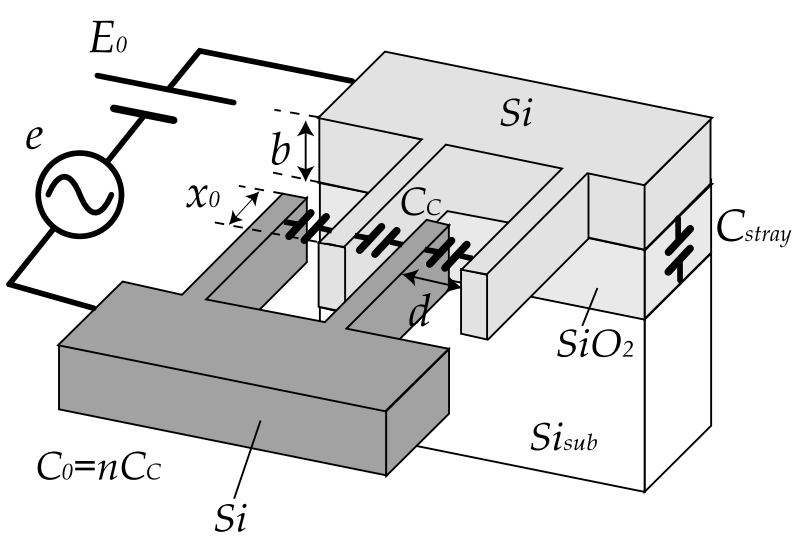

Fig. 1. Analysis model of a comb-drive actuator

$$
F=\frac{1}{2} r_{m} v^{2}+\frac{1}{2} R i^{2} \ldots \ldots \ldots \ldots \ldots \ldots \ldots
$$

Where $r_{m}$ is mechanical resistance, and $R$ is electrical resistance.

From the Lagrangian and the dissipation function, two Lagrange's equations can be then derived for the combdrive actuator applying $\mathrm{DC}$ bias $E_{0}$ and $\mathrm{AC}$ driving voltage $e$ :

$$
\begin{aligned}
f_{e}= & \frac{d}{d t}\left(\frac{\partial L}{\partial v}\right)-\frac{\partial L}{\partial x}+\frac{\partial F}{\partial v} \\
= & m \frac{d v}{d t}+r_{m} v+\frac{1}{C_{m}} \int v d t \\
& -\frac{1}{2}\left(\frac{q}{C(x)+C_{\text {stray }}}\right)^{2} \frac{d C(x)}{d x} \ldots \ldots . . . \\
E_{0}+ & e=R i+\frac{1}{C(x)+C_{\text {stray }}} \int i d t \ldots . . .
\end{aligned}
$$

where $f_{e}$ is an external force acting on the comb-drive actuators.

If we suppose the AC driving voltage $e$ is much smaller than $E_{0}\left(E_{0}>>e\right)$ having a single angular frequency $\omega$ and $f_{e}$ is synchronized with $e$, the equations are rewritten in complex forms in case of $R \approx 0$,

$$
\begin{aligned}
f_{a} & =j \omega m v_{a}+r_{m} v_{a}+\frac{v_{a}}{j \omega C_{m}}-A e_{a} \ldots \ldots \ldots \\
i_{a} & =j \omega\left(C_{0}+C_{\text {stray }}\right) e_{a}+A v_{a} \ldots \ldots \ldots \ldots
\end{aligned}
$$

where $C_{0}=\frac{2 n \varepsilon_{0} b x_{0}}{d}, A=\frac{C_{0} E_{0}}{x_{0}}$, and the suffix a denotes maximum amplitude of the each parameters.

Hence the equivalent circuit can be drawn as Fig. 2 for the comb-drive actuator. As shown in Fig. 2, the parameter $A$ is important factor to couple the electrical part with the mechanical part. If DC bias $E_{0}$ is zero, the coupling doesn't occur, while for large $E_{0}$ strong coupling is expected.

\section{Frequency Dependence on Admittance of a Comb-Drive Actuator}

As described the previous section, electrical and mechanical circuits are coupled when $D C$ bias $E_{0}$ is not zero. Therefore, it is possible to observe mechanical motion by measuring electrical characteristics. By solving

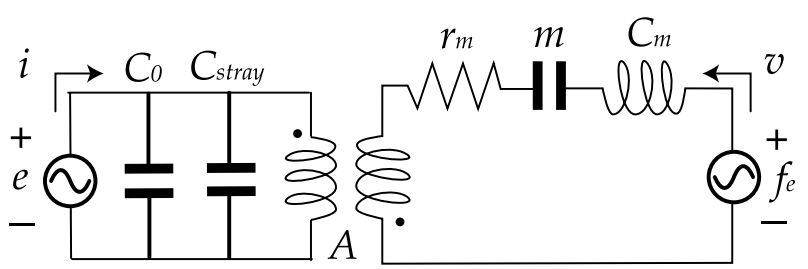

Fig. 2. The equivalent circuit of the comb-drive actuator

Eqs. 6 and 7 supposing $f_{a}=0$, we can obtain admittance function as follows:

$$
\begin{aligned}
Y(\omega) & =\frac{i_{a}}{e_{a}} \\
& =j \omega\left(C_{0}+C_{\text {stray }}\right)+\frac{A^{2}}{r_{m}+j\left(\omega m-\frac{1}{\omega C_{m}}\right)}
\end{aligned}
$$

The absolute value of the equation 8 , which can be measured by an impedance analyzer directly, is then given by

$$
\sqrt{\frac{A^{2}\left\{A^{2}-2 \omega\left(C_{0}+C_{\text {stray }}\right)\left(\omega m-\frac{1}{\omega C_{m}}\right)\right\}}{r_{m}^{2}+\left(\omega m-\frac{1}{\omega C_{m}}\right)^{2}}+\omega^{2}\left(C_{0}+C_{\text {stray }}\right)^{2}} .
$$

Fig. 3 shows a schematic representation of the function $|Y(\omega)|$. The admittance curve is on the line of a linear function $Y(\omega)=\omega\left(C_{0}+C_{\text {stray }}\right)$ at lower and higher angular frequencies, while shows one peak around the mechanical resonant frequency:

$$
\omega_{0}\left(=1 / \sqrt{m C_{m}}\right) \text {. }
$$

Note that the peak angular fequency $\omega_{p}$ is not corresponding to the resonannt angular frequency $\omega_{0}$ which is slighthly higher than $\omega_{p}$ since $\omega_{0}$ will be placed at the prolongation of the peak curve as shown in Fig. 3.

There is also a specific angular frequency $\omega_{1}$ determined by the following equation:

$$
A^{2}-2 \omega_{1}\left(C_{0}+C_{\text {stray }}\right)\left(\omega_{1} m-\frac{1}{\omega_{1} C_{m}}\right)=0
$$

This is the condition that the first term in the square root of Eq.9 becomes zero, hence $\omega_{1}$ can be plotted on the linear function $Y(\omega)=\omega\left(C_{0}+C_{\text {stray }}\right)$ in Fig. 3 . From Eq. 11, the relation between $\omega_{1}$ and $\omega_{0}$ is given by:

$$
\omega_{1}^{2}-\omega_{0}^{2}=\frac{A^{2}}{2 m\left(C_{0}+C_{\text {stray }}\right)} \cdots \ldots \ldots \ldots \ldots \ldots
$$

Since the right side of Eq. 12 always takes a positive value, we can know the relation $\omega_{0}<\omega_{1}$, consequently $\omega_{p}<\omega_{0}<\omega_{1}$. The strict relation between these angular frequencies will be discussed in the next section. 


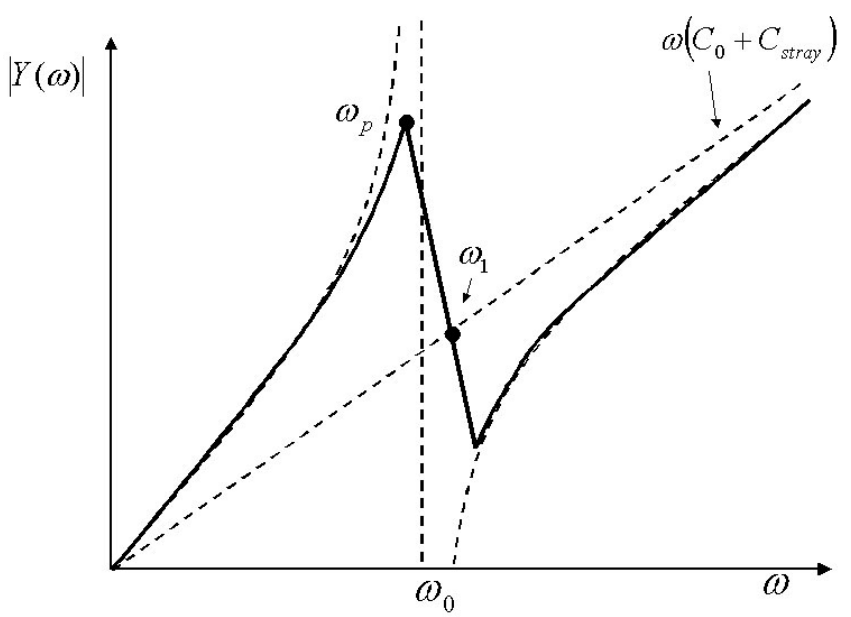

Fig. 3. Frequency dependence on absolute value of admittance

\section{Derivation of Device Parameters from Admittance Curve}

In order to obtain the relation between the device parameters and the specific angular frequency, we considered a function of $\omega^{2}$ which is made by subtracting $\omega^{2}\left(C_{0}+C_{\text {stray }}\right)^{2}$ from $|Y(\omega)|^{2}$ :

$$
\begin{aligned}
f\left(\omega^{2}\right) & =|Y(\omega)|^{2}-\omega^{2}\left(C_{0}+C_{\text {stray }}\right)^{2} \\
& =\frac{A^{2}\left\{A^{2}+\frac{2\left(C_{0}+C_{\text {stray }}\right)}{C_{m}}-2 m\left(C_{0}+C_{\text {stray }}\right) \omega^{2}\right\}}{r_{m}^{2}-\frac{2 m}{C_{m}}+m^{2} \omega^{2}+\frac{1}{\omega^{2} C_{m}^{2}}}
\end{aligned}
$$

Fig. 4 shows a typical variation of the $f\left(\omega^{2}\right)$ as a function of $\omega^{2}$. We can easily obtain $\omega_{1}^{2}$ as a point at which the function crosses the horizontal axis. As shown in Fig. 4, the graph has one peak and one bottom at the squared angular frequency of $\omega_{p}^{2}$ and $\omega_{b}^{2}$, respectively. By solving the equation $\frac{d f\left(\omega^{2}\right)}{d \omega^{2}}=0$, we can obtain $\omega_{p}^{2}$ and $\omega_{b}^{2}$ as follows:

$$
\begin{aligned}
& \omega_{p}^{2}= \frac{\omega_{0}^{4}-\omega_{0}^{2} \sqrt{\omega_{0}^{4}-\omega_{1}^{2}\left(2 \omega_{0}^{2}-\omega_{1}^{2}-\frac{r_{m}^{2}}{m^{2}}\right)}}{2 \omega_{0}^{2}-\omega_{1}^{2}-\frac{r_{m}^{2}}{m^{2}}} \\
& \omega_{b}^{2}=\frac{\omega_{0}^{4}+\omega_{0}^{2} \sqrt{\omega_{0}^{4}-\omega_{1}^{2}\left(2 \omega_{0}^{2}-\omega_{1}^{2}-\frac{r_{m}^{2}}{m^{2}}\right)}}{2 \omega_{0}^{2}-\omega_{1}^{2}-\frac{r_{m}^{2}}{m^{2}}}
\end{aligned}
$$

On the other hand, by making a tailor expansion of the $f\left(\omega^{2}\right)$ around $\omega_{0}^{2}$ and taking a first order approximation, the slope(denoted by a) at $\omega_{0}^{2}$ is given by:

$$
a=\frac{-2 m C_{0} A^{2}}{r_{m}^{2}}=\frac{-4 m^{2}\left(C_{0}+C_{\text {stray }}\right)^{2}\left(\omega_{1}^{2}-\omega_{0}^{2}\right)}{r_{m}^{2}}
$$

This can be rewritten in the following form:

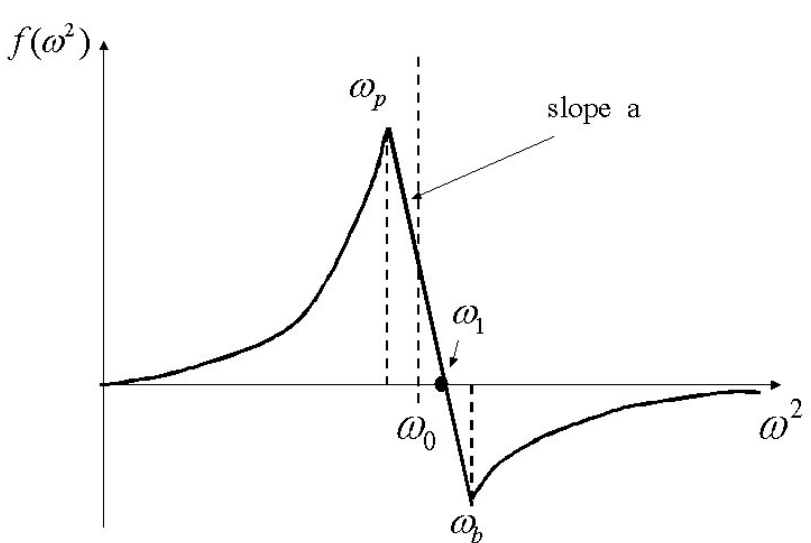

Fig. 4. Schematic diagram of the function $f\left(\omega^{2}\right)$

$$
\frac{r_{f}^{2}}{m^{2}}=-\frac{4\left(C_{0}+C_{s t r a y}\right)^{2}\left(\omega_{1}^{2}-\omega_{0}^{2}\right)}{a} \ldots \ldots \ldots
$$

Adding Eq. 14 to Eq. 15 and succesively substituting Eq. 17, we can obtain the expression of $\omega_{0}^{2}$ as follows:

$$
\begin{aligned}
& \omega_{0}^{2}=\frac{1}{4}\left\{\left(2-\frac{4\left(C_{0}+C_{\text {stray }}\right)^{2}}{a}\right)\left(\omega_{p}^{2}+\omega_{b}^{2}\right)\right. \\
& -\sqrt{\left.\left(\omega_{p}^{2}+\omega_{b}^{2}\right)\left\{\left(2-\frac{4\left(C_{0}+C_{\text {stray }}\right)^{2}}{a}\right)^{2}\left(\omega_{p}^{2}+\omega_{b}^{2}\right)+8\left(\frac{4\left(C_{0}+C_{\text {stray }}\right)^{2}}{a}-1\right) \omega_{1}^{2}\right\}\right\}}
\end{aligned}
$$

where the relation $\omega_{p}<\omega_{0}<\omega_{1}$ is used when driving this equation.

All of variables in the right side of Eq. 18 are measurable from the graph as shown in Fig. 4, consequently $\omega_{0}^{2}$ can be calculated. Once we find $\omega_{0}^{2}$, the moving mass $m$ can be estimated using Eq. 12, successively the compliance $C_{m}$ and the mechanical resistance $r_{m}$ are calculated by Eqs. 10 and 17, respectively. In this calculation, it is convenient to use a relarion $\frac{2}{\omega_{1}^{2}}=\frac{1}{\omega_{p}^{2}}+\frac{1}{\omega_{b}^{2}}$, which reduces one variable from Eq.18.

\section{Analysis of a Comb-Drive Actuator by Admittance Measurement}

As described in the previous sections, a comb-drive actuator can be analyzed by admittance measurement showing a peak related to the mechanical resonance. However, if the stray capacitance $C_{\text {stray }}$, which is mainly made between electrodes and a substrate, is much lager than the capacitance of opposing comb-drive fingers $C_{0}$, the peak height become very small. In the worst case, almost electrical current flow through the stray capacitance; as a result, no peak will be observed. Fig. 5 shows numerical admittance curves with the ratio $\frac{C_{\text {stray }}}{C_{0}}$ as parameter. As shown in the Fig. 5, the clear peak will be observed when $C_{0}$ and $C_{\text {stray }}$ are compatible. Therefore, we designed a comb-drive actuator with very small pad electrode as shown in Fig. 6.

When calculating the parameter $A$, a value of the gap $d$ is required. Since the gap usually become wider than the designed value due to undesirable under-etching, scan- 


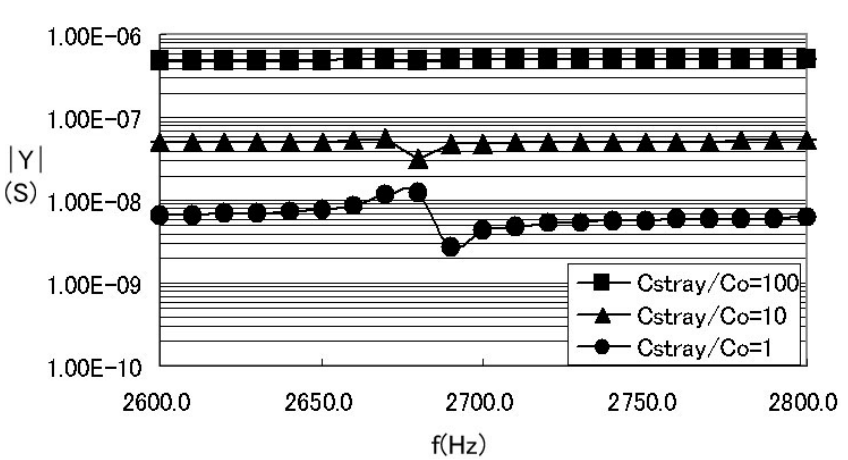

Fig. 5. Numerical admittance curves with the ratio $\frac{C_{\text {stray }}}{C_{0}}$ as parameter

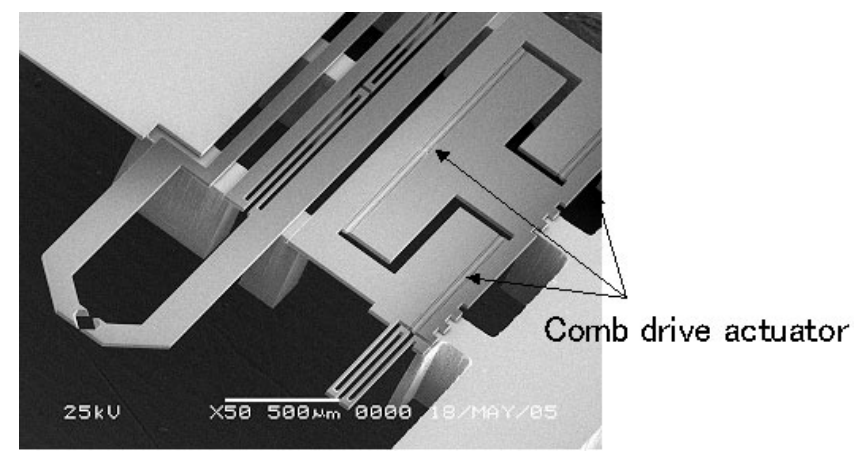

Fig. 6. A SEM photograph of the fabricated combdrive actuator

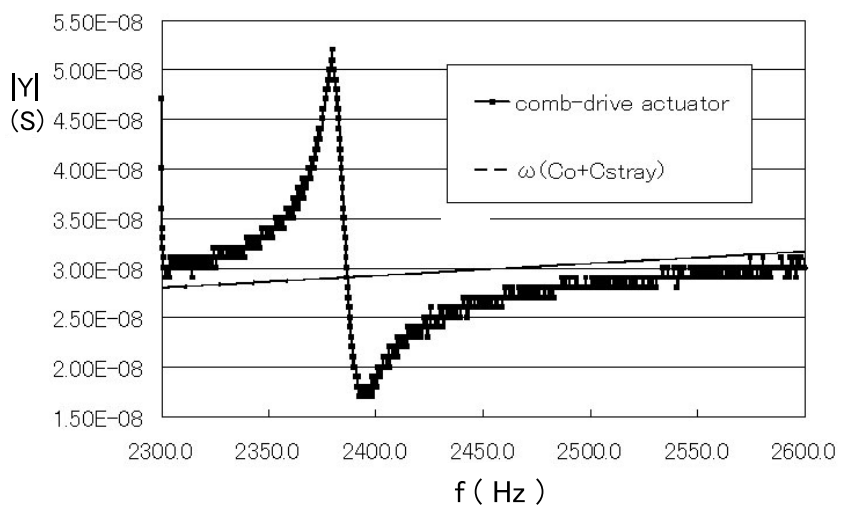

Fig. 7. Measured admittance as a function of $\omega$. DC bias and AC driving voltage are $17.5 \mathrm{~V}$ and 0.5 $\mathrm{Vpp}$, respectively. Total capacitance is approximately $2.00 \mathrm{pF}$

ning electron microscope (SEM) observation should be conducted in order to obtain correct value; for our fabricated device, $d$ was measured to be $3.50 \mu m$, then $A$ was calculated to be $5.53 \times 10^{-7} \mathrm{FV} / \mathrm{m}$.

Fig. 7 shows the admittance as a function of angular frequency measured by LCR meter (HP4192A) when DC bias and $\mathrm{AC}$ driving voltage are $17.5 \mathrm{~V}$ and $0.5 \mathrm{Vpp}$, respectively. The frequency step was $0.1 \mathrm{~Hz}$. By fitting the linear function, it was found that summation of $C_{0}$ and $C_{\text {stray }}$ was $2.00 \mathrm{pF}$.

Using this capacitance value, a graph of the function $f\left(\omega^{2}\right)$ was drawn as Fig. 8. From Fig. 8, the angular frequencies of $\omega_{1}, \omega_{p}, \omega_{b}$, and slope $a$ were determined to $1.4997 \times 10^{4} \mathrm{rad} / \mathrm{s}, 1.4951 \times 10^{4} \mathrm{rad} / \mathrm{s}, 1.5036 \times 10^{4} \mathrm{rad} / \mathrm{s}$,

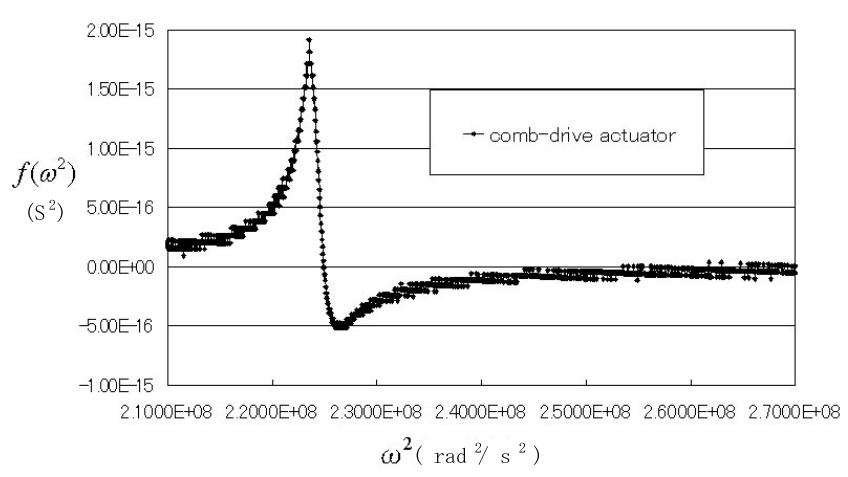

Fig. 8. The graph of the $f\left(\omega^{2}\right)$ converted from Fig. 7

Table 1. Measured device paremeters

\begin{tabular}{ccccc}
\hline$f_{0}(\mathbf{H z})$ & $m(\mathrm{~kg})$ & $C_{m}(\mathbf{m} / \mathbf{N})$ & $r_{f}(\mathrm{~kg} / \mathbf{s})$ & $\mathbf{Q}$ \\
\hline 2383.6 & $1.23 \times 10^{-7}$ & $3.62 \times 10^{-2}$ & $9.82 \times 10^{-6}$ & 188 \\
\hline
\end{tabular}

and $-1.56 \times 10^{-21} \frac{\mathrm{S}^{2}}{\mathrm{rad}^{2} / \mathrm{s}^{2}}$, respectively. The resonant frequency of the fabricated comb-drive actuator was hence found to be $2.3836 \mathrm{KHz}$ by substituting those value to Eq.18. Calculated device parameters are summarized in Table 1. The obtained moving mass showed good agreement with the mass which is estimated by the designed structure. However, the measured resonant frequency was smaller than the designed value $(3 \mathrm{KHz})$, eventually the compliance $C_{m}$ didn't agree with the designed one. We suppose the compliance is more sensitive to the fabrication process (especially undercut) than the mass due to cubed parameter dependency on the compliance.

\section{Conclusion}

We have described an analysis method of a comb-drive actuator by measuring admittance curve as a function of angular frequency. The admittance curve has a peak at frequency closing to the mechanical resonance. We showed an equation which can calculate resonant angular frequency from the admittance curve, and also introduced equations by which we can estimate device parameters such as mass, compliance, and mechanical resistance. The theory was examined by comparing experimental values with designed ones, and found that the presented method is useful to estimate mechanical parameters of a comb-drive actuator.

\section{Acknowledgment}

The authors would like to thank VDEC of the Univercity of Tokyo for providing masks of comb-drive actuators.

(Manuscript received Aug. 22, 2005, revised Jan. 19, 2006)

\section{References}

(1) H.A.C. Tilmans: "Equivalent circuit representation of electromechanical transducers: I. Lumped-parameter systems", J. Micromech. Microeng. Vol.6, pp.157-176 (1996)

( 2 ) C. Liang, F. Sun, and C.A. Rogers: "Electro-mechanical impedance modeling of active material systems", Smart Mater. Struct. Vol.5, pp.171-186 (1996) 
Kenjiro Ayano (Student Member) was born in 1976. He

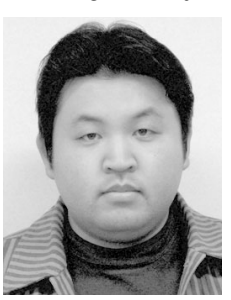
received his B.E. and M.E. degree from Tokyo University of Science, Yamaguchi in 1998 and 2000 , respectively. Since 2000, he has been at AOI ELECTRONICS., LTD. Currently, he is a Ph.D. student in the Graduate School of Faculty of Engineering, Kagawa University. He is engaged in the investigation of micro electro mechanical systems fabricated by semiconductor-based processes.

Katsuyori Suzuki (Student Member) was born in 1983. He

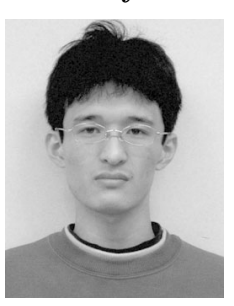
received his B.E. degree in Faculty of Engineering from Kagawa University. Curently, he is a M.E. student in the Graduate School of Faculty of Engineering, Kagawa University. He is engaged in the investigation of micro electro mechanical systems fabricated by semiconductor-based processes.
Gen Hashiguchi (Member) was born in 1963. He received

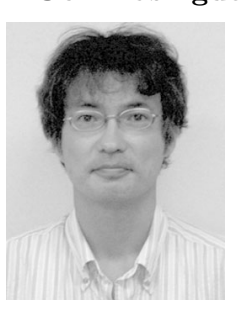
his M.S degree in electrical engineering from Chuo University, Tokyo, Japan, in 1988, and the Ph.D. degree in electrical engineering from The University of Tokyo, Tokyo, Japan, in 1996. From 1988 to 1998, he was with the Nippon Steel Corporation, where he was involved with the development of micro devices, including thermal sensors, field emitters, and amorphous silicon imaging sensors. From 1998 to 1999 , he was with the Japan Science and Technology Corporation(JST), as a Researcher of MEMS. He is currently a Professor of mechanical engineering at the Kagawa University, Kagawa, Japan. His current research interests are MEMS, with an emphasis on nanometer-scale mechanical devices and vacuum microelectronics.

Hiroyuki Fujita (Member) was born in 1952. He received

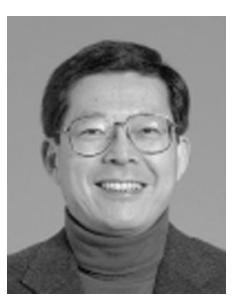
the B.S., M.S. and Ph.D. degrees in electrical engineering from the University of Tokyo, Tokyo, Japan in 1975, 1977 and 1980, respectively. He has been with the Institute of Industrial Science, the University of Tokyo as Lecturer (1980-81), Associate Professor (1981-93) and Professor (1993-present). He is currently a Director (2000-present) of Center for International Research on MicroMechatronics. He is engaged in the investigation of micro/nano electro mechanical systems fabricated by IC-based processes and applications to optics, bio and nano technology. He is also interested in autonomous distributed microsystems. He is a member of IEEE and IEEJ. 